\title{
Considerations on radar localization in multi-target environments
}

\author{
H. Rabe ${ }^{1}$, E. Denicke ${ }^{1}$, G. Armbrecht ${ }^{1}$, T. Musch ${ }^{2}$, and I. Rolfes ${ }^{1}$ \\ ${ }^{1}$ Leibniz Universität Hannover, Institut für Hochfrequenztechnik und Funksysteme, Appelstr. 9a, 30167 Hannover, Germany \\ ${ }^{2}$ Ruhr-Universität Bochum, Lehrstuhl für Elektronische Mess- und Schaltungstechnik, Universitätsstr. 150, 44801 Bochum, \\ Germany
}

\begin{abstract}
In a multitude of applications like e.g. in automotive radar systems a localization of multiple passive targets in the observed area is necessary. This contribution presents a robust approach based on trilateration to detect point scatterers in a two-dimensional plane using the reflection and transmission information of only two antennas. The proposed algorithm can identify and remove ambiguities in target detection which unavoidably occur in certain target constellations in such a two-antenna configuration.
\end{abstract}

\section{Introduction}

In recent years, a number of applications for close-range imaging via millimeter wave radar sensors like automotive radar systems (Wenger, 1998) or through-wall imaging (Yang and Fathy, 2007) emerged for locating multiple targets in the environment. Current implementations often use either mechanical shifts of the antenna position to synthesize a virtually enlarged aperture thus exhibiting an increased lateral resolution or use electronically adjustable narrow beam antenna arrays in order to detect the position of passive radar targets in two or three spatial dimensions. However, these approaches suffer from the complexity in hardware equipment and signal processing (Yang and Fathy, 2007). Instead of using Synthetic Aperture Radar (SAR) or beam-steerable imaging, this contribution presents an approach related to the one described in Michael et al. (2000) which features a reduced system complexity by only using the information acquired from two fixed antennas for the detection of multiple scatterers in a plane. The wanted position information is reconstructed out of the complex reflection and transmission scattering parameters obtained over a certain bandwidth. The reconstruction is done by first extracting the dominant scat-

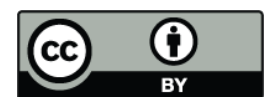

Correspondence to: H. Rabe

(rabe@hft.uni-hannover.de) tering centers out of all three data sets separately via Prony's method (Carriere and Moses, 1992) and combining them in a trilateration algorithm to allocate the scatterers according to their position in a plane. The advantage of this approach is the possibility to detect or even to remove ambiguities which inevitably appear as so called "ghost" targets (Helmbrecht and Biebl, 2005) in certain geometrical constellations of targets and antennas.

The paper is organized as follows. Section 2 describes the basic principle of point target reconstruction with the proposed trilateration approach. That section derives the accuracy in terms of the ambiguity area and presents a systematic approach for determining the occurrence of ghost targets. The Sect. 3 describes the implementation of the target reconstruction algorithm. Some characteristic simulation results are presented in Sect. 4 to confirm the expected properties of the algorithm using synthesized data sets. The paper ends with a conclusion.

\section{Properties of point target reconstruction}

The trilateration procedure for reconstructing point targets from the reflection and transmission scattering data of two antennas is done according to Fig. 1. The two transmit and receive antennas are situated on the $\mathrm{x}$-axis of a local coordinate system at the positions $x_{1}=-20 \lambda_{c}$ and $x_{2}=20 \lambda_{c}$, where $\lambda_{c}$ is the free-space wavelength at center frequency. The antennas ant $t_{1}$ and ant $_{2}$ are assumed to radiate in the front half space which is valid for many applications so that the shaded area in the figure can be neglected. A point scatterer is situated at position $x=0 \lambda_{c}$ and $y=20 \lambda_{c}$ and is illuminated by antenna 1 and 2 . The complex scattering parameters are extracted over the bandwidth $B$. The next step is to extract the Time Of Flight (TOF) information to the scatterer for both reflections and the transmission in order to calculate the distances $r_{1}, r_{2}$ and $t_{21}$. The propagation velocity is assumed to be the speed of light $c_{0}$. The TOF estimation can be done in

Published by Copernicus Publications on behalf of the URSI Landesausschuss in der Bundesrepublik Deutschland e.V. 


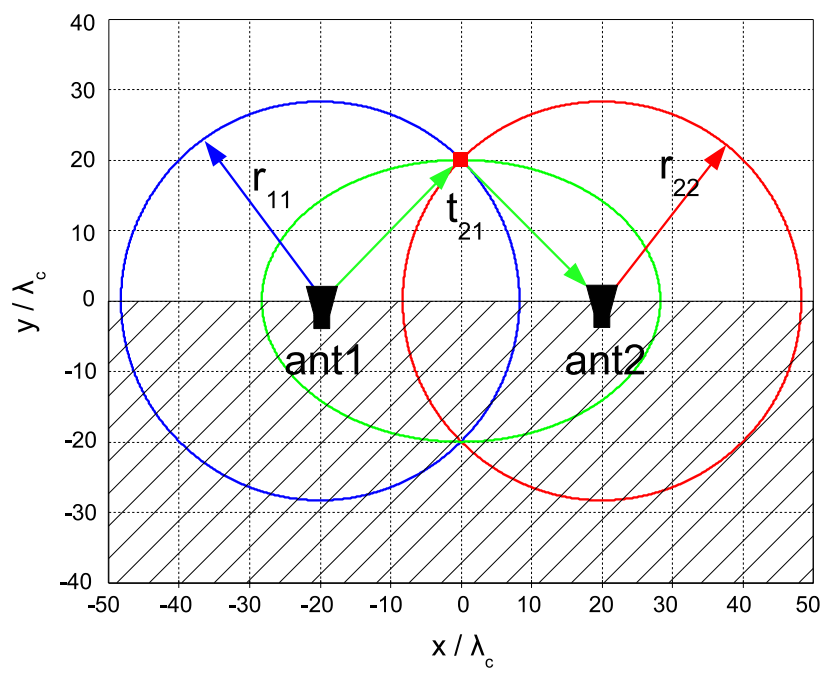

Fig. 1. Trilateration algorithm exploiting transmission and reflection information.

several ways. We used Prony's method (Carriere and Moses, 1992). As depicted in Fig. 1 the point target can be reconstructed in the xy-plane by determining the intersection point between the two circles which determines the possible target position using the reflection data and the ellipse resulting from the TOF of the transmission signal ${ }^{1}$.

In the following Sect. 2.1 the ambiguity area of a point target is derived in dependence of its position which is a direct measure for the precision of the reconstructed target location. Besides the ambiguity of the target position within a certain area other ambiguities arise in a scenario of multiple point scatterers. These ambiguities are called ghost scatterers. In Sects. 2.2 and 2.3 this kind of ambiguity will be examined. In some cases these ambiguities can be removed or at least be detected which will be explained in Sect. 3 .

\subsection{Ambiguity area of a single scatterer}

The reconstruction accuracy of a point scatterer using the trilateration method depends on the SNR (Signal to Noise Ratio) of the collected data. The noise power causes an uncertainty in the estimation of the TOF values. Assuming a distance uncertainty of $2 \Delta r_{\mathrm{amb}}$ applied to both reflection and transmission data the intersection point expands to an area wherein the real scatterer position is located. The ambiguity area for a scatterer directly placed in front of the antennas is shown in Fig. 2. The closest blue circle results from the real scatterer position and the second one is the result obtained with an uncertainty $2 \Delta r_{\mathrm{amb}}$. By applying the same uncertainty on the right antenna four intersection points will occur between the two blue and the two red circles marking the

\footnotetext{
${ }^{1}$ The sum of the distances from ant ${ }_{1}$ to the scatterer and back to $a_{2}$ is constant when the scatterer is placed on an ellipse where the antennas correspond to its focal points.
}

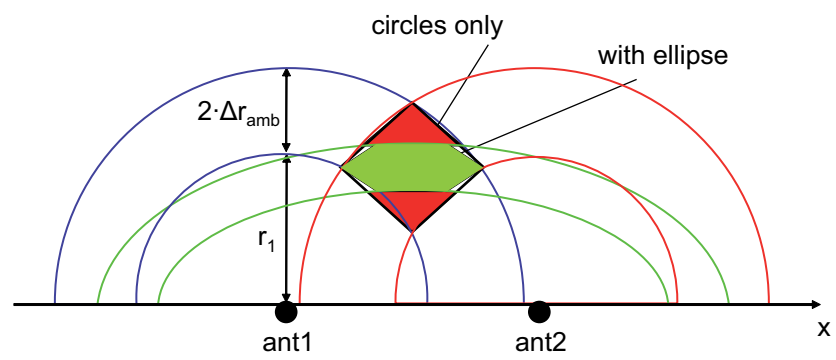

Fig. 2. Ambiguity area for intersection of circles only and in combination with transmission ellipses with radial uncertainty of $2 \Delta r_{\mathrm{amb}}=0.1 \lambda_{c}$.

edges of a rhombus which is approximately equivalent to the ambiguity area. The area consists of two red triangles and the hexagon in the middle of the area. Considering the transmission information with the same uncertainty of $2 \Delta r_{\mathrm{amb}}$ the ambiguity area is shrinked to the green hexagon. As mentioned before, the area is only an approximation which gets worse in target positions near the $\mathrm{x}$-axis due to the flat angles between the intersection of the red with the blue circles.

To get an impression on the expected precision for target reconstruction the ambiguity area has been examined in dependence on the scatterer position in the xy-plane. Assuming an uncertainty of $2 \Delta r_{\mathrm{amb}}=0.1 \lambda_{c}$, the values for the ambiguity area $\mathrm{A}$ related to $\lambda_{c}^{2}$ are given in Fig. 3. Due to the symmetry of the scenario only the results for positive values of $\mathrm{x}$ are displayed. The antennas are placed as before at $x_{1}=-20 \lambda_{c}$ and $x_{2}=20 \lambda_{c}$. The lines of same ambiguity area size are highlighted by the gradation. The lines of same area size exhibit shapes similar to ellipses. With increasing distance of the scatterer in $\mathrm{x}$ - and y-direction the ambiguity area enlarges. Especially for scatterers close to the $\mathrm{x}$-axis the ambiguity area can reach big values. Therefore the proposed system works best in small distances from the antenna position and in noticeable distance from the $\mathrm{x}$-axis. A further analysis has shown that a larger antenna distance leads to smaller ambiguity areas. This is obvious since a variation of the antenna distance can be regarded as a scaling of the scenery. At first sight it seems to be the best to separate the antennas in order to benefit from the shrinking ambiguity areas. However, with increasing antenna distance also the probability of ghost scatterers increases as shown in the following section.

\subsection{Ambiguity in multi-target environment}

Besides the ambiguity area of one scatterer another ambiguity arises by the presence of more than two point scatterers. The reconstruction results in a special constellation of three scatterers in a row along the y-axis is shown in Fig. 4. The figure shows some of the circles and ellipses that occur after processing the scattered signals. The real scatterers $s_{1}, s_{2}$ and $s_{3}$ are highlighted red and the ghost scatterers are marked 


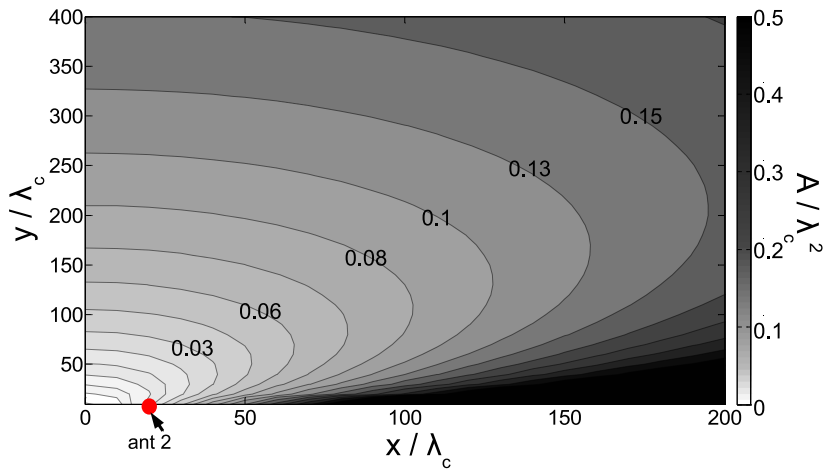

Fig. 3. Ambiguity area size in dependence of scatterer position with a radial uncertainty of $2 \Delta r_{\mathrm{amb}}=0.1 \lambda_{c}$.

green. The blue circle stands for the TOF between target $s_{1}$ and antenna 1 with the radius $r_{11}$. The first index denotes the target number and the second one is the antenna index. The circles $r_{11}, r_{32}$ and the transmission $t_{2}$ (transmissions have a target index only due to channel reciprocity) generate two ghost targets on the $\mathrm{x}$-axis. The occurrence of ambiguities depends on the scatterer distances as seen from the antennas. The condition in Eq. (1) for the radial distances of the scatterers from antenna 2 must be met to generate ambiguities on the $\mathrm{x}$-axis.

$r_{12}=r_{11}$

$r_{32}=r_{11}+d_{\mathrm{ant}}$

$r_{22}=\frac{t_{2}}{2}=r_{11}+\frac{d_{\mathrm{ant}}}{2}$

The first scatterer distance can be arbitrarily chosen on the $y$-axis. Due to the symmetry of the problem the radius $r_{12}$ is the same as $r_{11}$. If the radial distances of the following two scatterers increase in steps of $d_{\text {ant }} / 2$ two ghost targets will occur symmetrically to the y-axis on the x-axis. Decreasing the radial distance below $d_{\text {ant }} / 2$ causes the intersection point to move to positive values of $y$. Increasing the distance will otherwise cause no more intersections and therefore the generation of ghost targets will be avoided. This statement is also valid for other scatterer constellations generating a ghost target on the $\mathrm{x}$-axis. These constellations can be achieved by moving the scatterers along the circles and the ellipse in Fig. 1. All these constellations offer at least one radial distance between the scatterers and the antennas that is closer than $d_{\text {ant }} / 2$, so the minimal radial distance of the scatterers is $d_{\text {ant }} / 2$ to avoid ambiguities and can be achieved by aligning the scatterers on the y-axis. All other constellations leading to ghost targets above the $\mathrm{x}$-axis need a radial spacing of the scatterers of less than $d_{\mathrm{ant}} / 2$ so ghosts can be completely avoided by scatterer spacing larger than $d_{\mathrm{ant}} / 2$. If the transmission and reflection signals allow a reconstruction in range cells with the size of $d_{\text {ant }} / 2$ or larger there would not be an ambiguity. Utilizing for example a Fourier transform to get the band-limited impulse response will result in the well

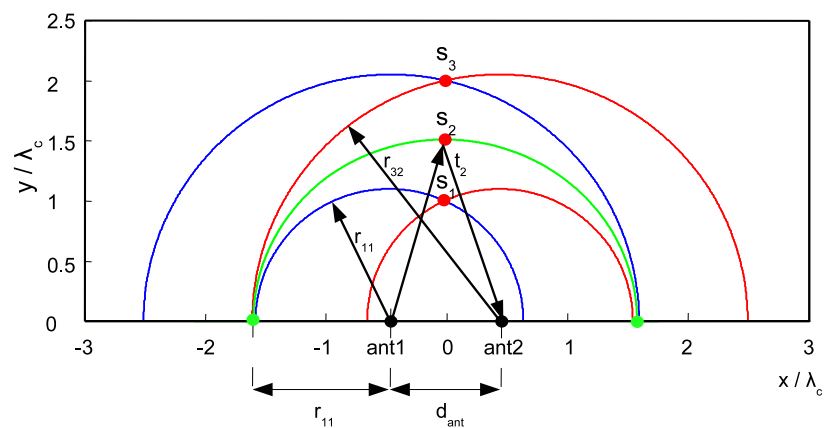

Fig. 4. Ghost scatterers resulting from intersections between two circles and one ellipse from three real scatterers.

known resolution cells that have a minimum size depending on the bandwidth $\mathrm{B}$ and the propagation velocity $c_{0}$ according to Eq. (2).

$\Delta r_{\text {res }}=\frac{c_{0}}{2 B}$

To avoid the occurrence of ambiguities the value of $\Delta r_{\text {res }}$ has to be larger than $d_{\text {ant }} / 2$ according to the preceding explanations. This relationship is shown in Eq. (3) and can be regarded as a design criterion for the antenna distance for a given signal bandwidth to suppress ghosts completely.

$d_{\text {ant }} \leq \frac{c_{0}}{B}$

The expression can vary with different window functions in the frequency domain before applying the Fourier transform or with the beamwidth of the used antennas. The window function causes an increase of the size of a resolution cell. By reducing the beamwidth to less than in the omnidirectional case the ambiguities occurring in the area which is not covered by the antenna's beam can be removed by plausibility. Equation (3) can therefore be regarded as a worst case without windowing and applying an omnidirectional beam.

\subsection{Higher order ambiguities}

It was shown how ghost targets arise and how they can be avoided. However, in some cases the choice of a small antenna distance to avoid such ambiguities probably does not fulfill the requirements of reconstruction accuracy, as a small antenna distance leads to bigger ambiguity areas as explained in the preceding Sect. 2.1. If the number of scatterers is known in a certain application, an upper bound for the number of ghost scatterers can be determined that can assist the reconstruction algorithm to limit the detected scatterers to a physically plausible value as explained in Sect. 3. By adding one more target to the scene in Fig. 4 on the y-axis in a distance less or equal to $d_{\mathrm{ant}} / 2$ from target $s_{3}$ relative to the antenna positions, further two ambiguities will be generated symmetrically to the y-axis. The situation gets more complicated if the number of scatterers approaches five or more 


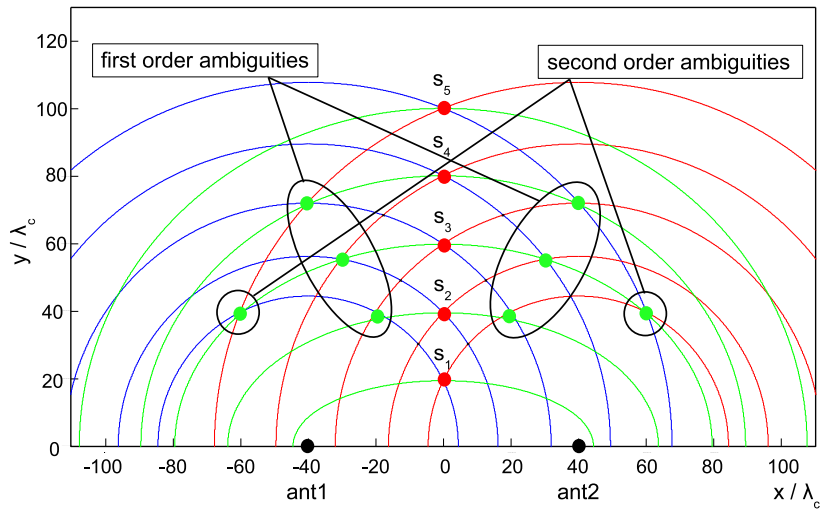

Fig. 5. Occurrence of higher order ghost scatterers in a scenario of five real scatterers.

and the distance between the first and the last scatterer stays $d_{\text {ant }} / 2$. In the case of five scatterers not only the neighboring scatterers cause ambiguities as shown in Fig. 5. Besides the expected ambiguities formed by the adjacent scatterers that now move to positive $y$-values due to the smaller scatterer distances there will occur two more ambiguities named "second order" ambiguities. These new ghosts are formed by the information $r_{11}, t_{3}$ and $r_{52}$ which now satisfy the condition of a distance smaller than $d_{\mathrm{ant}} / 2$ and therefore lead to ambiguities as in the three scatterer scenario above occurring again on the X-axis. It can be shown easily that seven scatterers lead to "third order" scatterers, nine scatterers lead to "fourth order" scatterers and so on if the radial distances between the outer scatterers stay below $d_{\text {ant }} / 2$. Assuming no restriction in the system's resolution an upper limit for the ghost scatterers $N_{g}$ can be derived in dependence of the number of the real scatterers $N_{S}$ and the maximal order $O$ of the scenario according to Eq. (4).

$$
\begin{aligned}
N_{g} & =2 \cdot \sum_{n=3}^{2 O+1}\left(N_{s}+1-n\right) \text { for } n=3,5, \ldots, 2 O+1 \\
O & =\frac{d_{\mathrm{ant}}}{2 x_{s}}
\end{aligned}
$$

The maximal order $O$ is the largest uneven value in $d_{\mathrm{ant}} / 2 x_{s}$ where $x_{s}$ is the radial distance between neighboring scatterers. This formula is valid for equally spaced scatterers aligned on the y-axis with a minimal distance of $d_{\text {ant }} / 2$ between the nearest and the farthest scatterer and is an upper limit for the occurring ambiguities. The order can be reduced by lowering the antenna distance or increasing the scatterer distance. In Sect. $4 N_{g}$ is displayed for maximal order in Fig. 8 up to $N_{s}=15$. The number of ghost scatterers is increasing exponentially with higher values of $N_{s}$. In Sect. 4 the benefit of knowing the upper limit for ambiguities is discussed in order to reduce the number of detected targets.

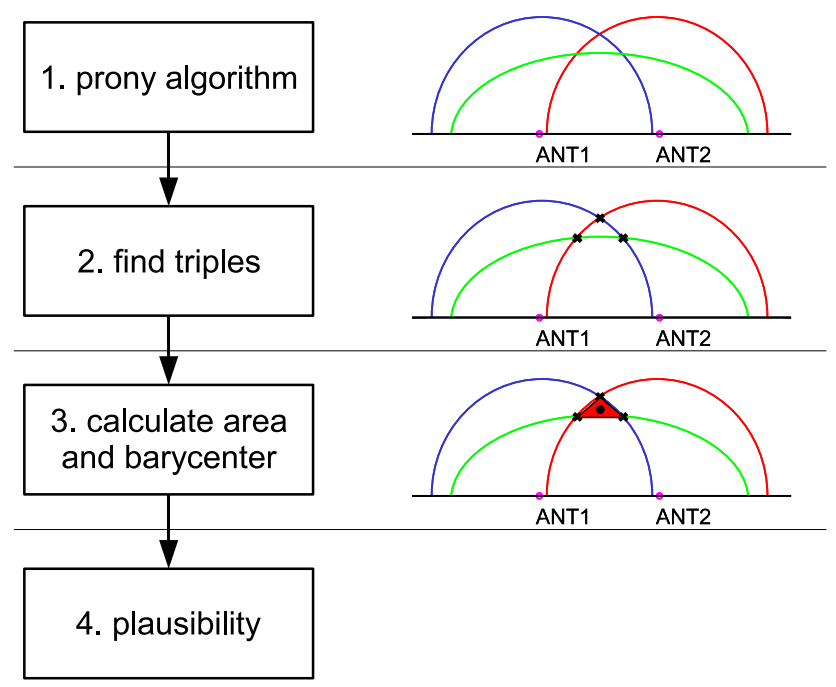

Fig. 6. Algorithm steps for point target reconstruction in a plane.

\section{Algorithm}

The results from the previous section have been integrated as a part of the presented algorithm for point target reconstruction. The single steps of the algorithm are shown in Fig. 6. As an example, the reconstruction is explained for one point scatterer. At first the complex scattering parameters in the frequency domain of the two reflections and the transmission are inserted into a Prony algorithm according to Carriere and Moses (1992). This parametric approach directly extracts the scatterer distances for the three parameter sets. The parameters are obtained by approximating the signal in the frequency domain as a sum of weighted exponential functions corresponding each to a single scattering center. The approximation is done in a least squares sense. The Prony's method offers good results at high SNR. At low SNR (20 dB and lower) however, other algorithms like peak detectors applied on the time domain signal obtained by a Fourier transform have offered more robustness. In the given example the reflection and the transmission information will each contain one value corresponding to the only scatterer in the scenario. These values are inserted into an analytical expression calculating the intersection points between the blue circles from antenna 1 and the transmission ellipse. In contrast to the reconstruction using perfect data the intersection points of all three geometries do not hit a single point but will expand to three points accordingly as displayed in the figure when using noisy data. Hence, the corresponding triples belonging to one point scatterer have to be found in step 2 using a nearestneighbor method. Furthermore, a barycenter is calculated out of the spanned triangle as an approximation for the real target position in step 3. Due to the imperfections of point scatterer reconstruction it is sometimes not possible to find the correct triple of one scatterer using the nearest-neighbor 


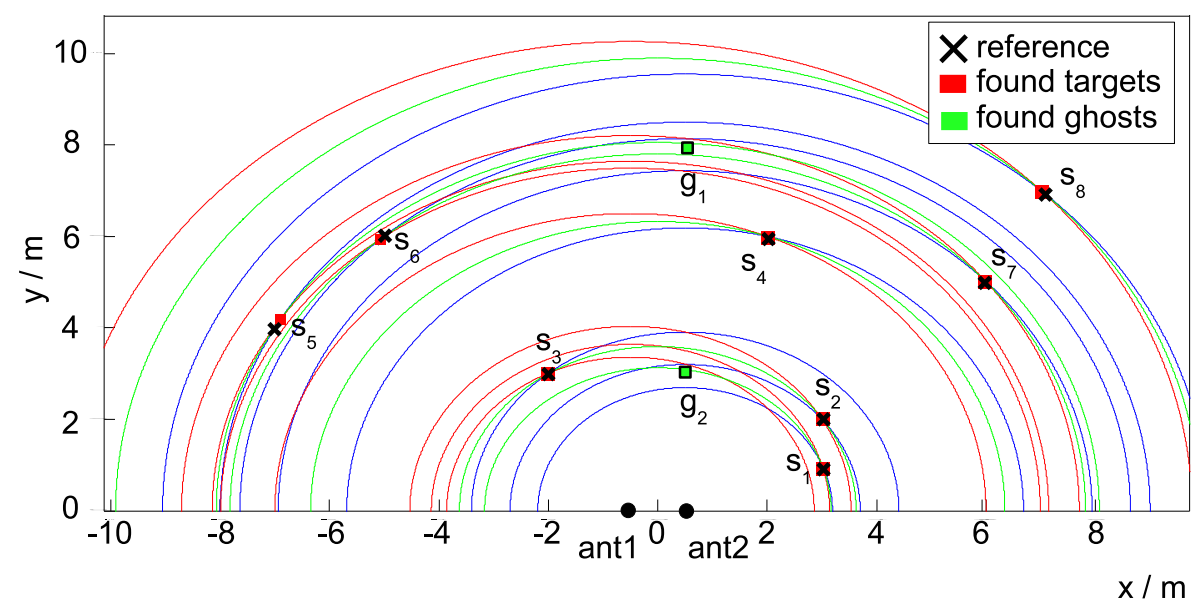

Fig. 7. Example of the reconstruction of 8 scatterers using the trilateration method with plausibility check.

method. Likewise the probability to detect ghost scatterers increases as an exact intersection of both circles and the ellipse is no more necessary for a scatterer detection. Therefore a plausibility check is performed at the end of the algorithm in step 4.

If the number of the found triangles is larger than the sum of the upper limit for the number of ghost scatterers (see Eq. 4) plus the number of real scatterers (the number of real scatterers is given by the number of targets detected by the Prony algorithm) the algorithm has detected more scatterers than physically possible for the current scenario. The algorithm therefore removes the biggest triangles until the upper limit is reached. A further reduction can be achieved by removing triangles with ambiguous information. If a triangle is constructed by either a circle or an ellipse only crossing a single scatterer then the point belonging to the spanned triangle is unambiguous. An example is depicted in Fig. 4 where the ghost targets on the $\mathrm{x}$-axis consist of the circles and the ellipse of the real targets. The real targets all have at least one circle or ellipse that is uniquely used for their scatterer reconstruction and can therefore be marked as "real" target. This information allows to identify real scatterers in the whole pool of possible scatterers. This principle does not work with all configurations as the position of a ghost target can for example coincide with a real scatterer which is then marked as ghost target. The next section shows some simulation results showing the detection capability of the algorithm.

\section{Simulation results}

To verify the capability of target reconstruction using the trilateration algorithm a scenario with 8 scatterers is chosen which are placed in a rectangular field of the size $20 \mathrm{~m} \times 10 \mathrm{~m}$. In Fig. 7 the 8 scatterers are placed at the positions marked as black crosses. The antennas are placed in a distance of $1 \mathrm{~m}$ symmetrical to the origin. The scatter- ing parameters have been determined over a bandwidth of $2 \mathrm{GHz}$. To obtain realistic values a white noise signal was added to the scattering data. The result for an SNR of $35 \mathrm{~dB}$ is shown by the red and green rectangles which mark the reconstructed scatterer positions. The red rectangles have at least one unique information so they can be regarded as real scatterers. The green rectangles mark the scatterers which only use information that is already used for other scatterers. The scatterer $g_{2}$ ( $g$ stands for "ghost") for example is generated by the ellipse from $s_{1}$, the blue circle from $s_{2}$ and the red circle from $s_{3}$. As explained in Sect. 2.2 the ambiguities originate from a radial scatterer distance of less than $d_{\text {ant }} / 2$ which is true for the scatterers $s_{1}, s_{2}$ and $s_{3}$. The same situation leads to the ghost $g_{1}$ which is formed by the scatterers $s_{5}, s_{6}$ and $s_{7}$. Although these scatterers have a large distance among each other, they are separated very closely as seen from the antennas. In contrast the scatterers $s_{4}$ and $s_{8}$ are separated with a higher radial distance so they do not generate ambiguities. The figure also gives a hint regarding the accuracy of the reconstruction. As shown in Sect. 2.1 the accuracy degrades with higher radial distance to the antennas as can be seen for scatterer $s_{8}$ where the rectangle is a slightly shifted out of the reference position. Another degradation can be seen at scatterer $s_{5}$ at approximately the same radial distance but a position closer to the $\mathrm{x}$-axis. These conditions lead to a stronger deviation of the reconstructed point to the reference position due to the larger ambiguity area.

In the example of Fig. 7 the number of ghost scatterers is much less than the theoretical limit of 24 as is shown in Fig. 8. The black curve shows the relation between the number of scatterers $N_{s}$ and the maximum number of ghosts $N_{g}$ for this case. The blue and the red curve are the results for the found ghosts by placing the scatterers randomly in the scenery for two different adjustments in the algorithm. By regarding the deviation of both curves from the black curve it is obvious that the theoretical limit of ghost scatterers is 


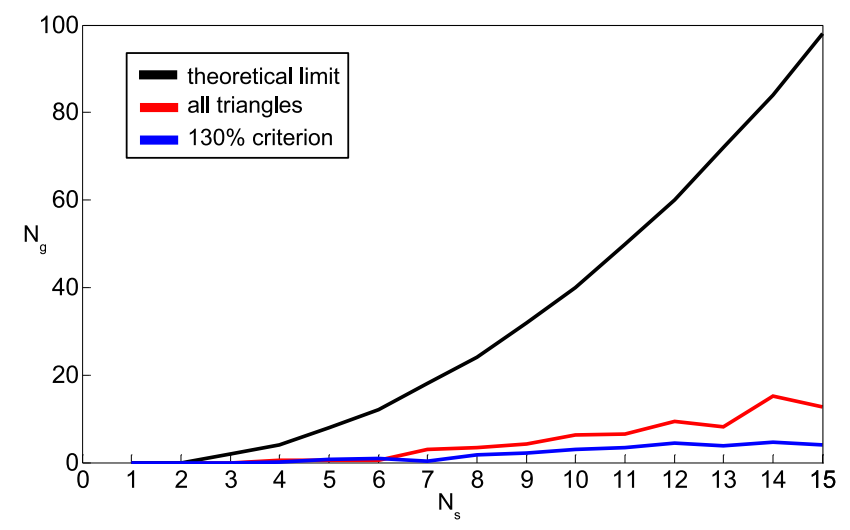

Fig. 8. Number of ghost targets in dependence of real scatterers.

seldom reached in scenarios with randomly spaced scatterers. For this reason the theoretical limit is not a reasonable choice for reducing the number of triangles found by the algorithm as expected in Sect. 2.3. The red curve shows the result of the algorithm by considering all triangles found in the nearest-neighbor search and is significantly lower than the theoretical limit. In order to reduce the ambiguities due to the number of triangles found by the nearest-neighbor algorithm beside the real scatterers and the ghosts, a limit for the number of triangles has been introduced to $130 \%$ of the scatterers resolved by the Prony algorithm. Only the smallest triangles are considered which reduces the number of ghost scatterers. The value of $130 \%$ has been found to be a good compromise between the number of reconstructed ghost targets and the certainty of reconstructing all physically available scatterers.

The simulations showed that the algorithm detects ghost targets as expected. Due to the generation of further ambiguities by the search for triangles it is useful to reduce the number of detected scatterers. The proposed algorithm showed good results with a fixed limit of the resolved triangles.

\section{Conclusions}

A trilateration algorithm was introduced that reduces the problem of ghost scatterers in a two-antenna setup for imaging purposes in the plane. The basis of this approach is the systematic description of the generation of ghost targets in dependence of the scatterer placing and the choice of the antenna distance. It can be concluded that a two-antenna setup provides imaging in a tradeoff between reconstruction precision and the occurrence of ghost targets. It was shown that by reducing the system's resolution and under-run a certain antenna distance on the one hand leads to a complete elimination of ghosts. On the other hand a possibility of detecting ghost scatterers was presented that provides a save detection of at least some of the real targets in the scenery without limitation in resolution or antenna distance allowing the enhancement of the reconstruction accuracy by choosing high antenna distances.

\section{References}

Carriere, R. and Moses, R.: High resolution radar target modeling using a modified Prony estimator, IEEE T. Antenn. Propag., 40, 13-18, 1992.

Helmbrecht, E. and Biebl, E.: Radar imaging using noncoherent sensors, Proc. IEEE MTT-S International Microwave Symposium Digest, 4 pp., 2005.

Michael, B., Menzel, W., and Gronau, A.: A real-time close-range imaging system with fixed antennas, IEEE T. Microw. Theory, 48, 2736-2741, 2000.

Wenger, J.: Future Trends in Automotive Radar/Imaging Radar, Proc. 28th European Microwave Conference, 1, 636-664, 1998.

Yang, Y. and Fathy, A.: Design and Implementation of a Low-Cost Real-Time Ultra-Wide Band See-Through-Wall Imaging Radar System, Proc. IEEE/MTT-S International Microwave Symposium, 1467-1470, 2007. 